\title{
DELAYED BILATERAL HYDROTHORAX AFTER CENTRAL VENOUS CATHERIZATION: A CASE REPORT
}

\author{
Arpana J1', Moses Charles D’souza², Kutappa A. M³ , Deepa Baskaran ${ }^{4}$
}

\section{HOW TO CITE THIS ARTICLE:}

Arpana J, Moses Charles D’souza, Kutappa A. M, Deepa Baskaran. "Delayed Bilateral Hydrothorax after Central Venous Catherization: A Case Report". Journal of Evolution of Medical and Dental Sciences 2014; Vol. 3, Issue 46, September 22; Page: 11310-11313, DOI: $10.14260 /$ jemds/2014/3477

ABSTRACT: Central venous catheterization is a common procedure in anesthetic management of patients undergoing major surgery or care of critically ill patients. Delayed complication such as hydrothorax, hydromediastinum or cardiac tamponade is extremely rare with a few case reports. We report a case of bilateral hydrothorax due to migration of the tip of the central venous catheter from within the vein into the mediastinum following subclavian vein catheterization.

KEY WORDS: Central venous catheterization, bilateral hydrothorax, total parental nutrition.

INTRODUCTION: Central venous catheterization is a useful procedure for intra operative hemodynamic monitoring, for infusion of large volume of fluid, difficult peripheral venous access and parental feeding. However, it can cause various complications such as pneumothorax, haemothorax, air embolism, intravascular thrombus which are simple to explain with our current knowledge of anatomy. As the need for long term catheterization increases for reasons such as total parenteral nutrition (TPN), the probability of delayed complications including hydrothorax, hydromediastinum increases. $^{1}$

Reports of ipsilateral pleural effusion following misplacement of catheter are not unusual. However, simultaneous occurrence of bilateral pleural effusion probably due to communication between the two pleural cavities is extremely rare with only a few case reports. Hence we report a case of bilateral pleural effusion after performing central venous catheterization due to catheter tip migration and abnormal location.

CASE REPORT: A 26 year old man was referred to our hospital for management of abdominal sepsis ten days ago patient had undergone a explorative laparotomy for a pancreatic fistula resulting from a blunt injury abdomen.

A repeat laparotomy with drainage of 100 milliliter $(\mathrm{ml})$ of pus and peritoneal lavage was done. On the second postoperative day in view of high output enterocutaneous fistula, it was decided to put the patient on TPN.

A right subclavian triple lumen central venous catheter was inserted by an experienced resident using percutaneous seldingers technique and good back flow in all three ports. A portable anteroposterior chest radiograph confirmed the position of the catheter to be satisfactory. Two days after the commencement of TPN, patient was found to have dyspnea and tachypnea. High flow oxygen via face mask was needed to keep his arterial oxygen saturation above $90 \%$.

Hence patient was shifted to intensive care unit for management. On admission his heart rate was $110 \mathrm{~b} / \mathrm{min}, \mathrm{BP}-100 / 60 \mathrm{mmHg}$, SPo2 on 6L, 02 was 98\%. Arterial blood gas analysis showed pH - 7.27, Pco2 - 59 mmHg, Po2 - 104 mmHg, Hco3 - 24.6. 
In view of severe tachypnea (RR - $38 \mathrm{bpm}$ ) and impending respiratory failure, it was decided to intubate and mechanically ventilate the patient. Patient was induced through the proximal post of right SCV catheter with Inj. Fentanyl 140mg, Inj. Midazolam $3 \mathrm{mg}$ IV and Inj. Succinyl choline 100mg IV, 1 liter Crystalloid (Normal Saline) and TPN infusion was continued.

Two hours after intubation and mechanical ventilation, maximum inspiratory pressure rose from $23 \mathrm{~cm} \mathrm{H} 2 \mathrm{O}$ to $38 \mathrm{~cm} \mathrm{H} 2 \mathrm{O}$. On auscultation crackles were present. Assuming, that increased airway pressure was due to increased secretions, endotracheal aspiration was attempted but almost no discharge was found. Tidal volume was reduced to $8 \mathrm{ml} / \mathrm{kg}$ from $10 \mathrm{ml} / \mathrm{kg}$ and arterial blood gas and chest radiograph were repeated. ABG showed $\mathrm{pH}$ of $7.43, \mathrm{PaCO} 2-40 \mathrm{~mm}, \mathrm{PaO} 2-135 \mathrm{mmHg}$, HCO3 - 25.3 and chest X ray showed bilateral massive pleural effusion.(Figure:1) Ultrasound scan of the thorax confirmed the presence of bilateral large pleural effusions.

A 28 french intercostal chest drain was placed into each side of the chest. $800 \mathrm{ml}$ and $450 \mathrm{ml}$ of white, milky blood stained fluid was drained from right and left side immediately. As the fluid was suspected to contain TPN due to its color and properties, a check aspiration through CVC was carried out to reconfirm the position of the catheter. Blood was aspirated in the proximal but not the distal catheter port. Assuming that a possibility of the abnormal location of catheter tip caused the hydrothorax, CVC was removed and a new central venous catheter was installed under ultrasound guidance through internal jugular vein on right side.

Biochemical analysis of the drained fluid from both sides suggested that it was TPN. Two samples of drained fluid had glucose concentrations of $280 \mathrm{mg} / \mathrm{dl}$ and $230 \mathrm{mg} / \mathrm{dl}$. The patients corresponding blood glucose was $90 \mathrm{mg} / \mathrm{dl}$ at the time. The glucose concentration of TPN was 2.7 $\mathrm{g} / \mathrm{dl}$. The drain fluid amylase was 60 while Serum amylase was 319 ruling out the possibility of pancreatic effusion. The drain fluid was possibly a mixture of crystalloid, TPN and pleural fluid.

Over two liters of fluid was drained from both sides over the next 4 days, after which ABG showed a pH 7.40, PCO2 36, Po2 - 103, with an Fio2 of 40\%. The chest radiographs showed resolution of bilateral pleural effusions and re-expansion of both lungs.(Figure:2). Antibiotic treatment with piperacillin -Tazobactum and Metronidazole was continued. Over the next one week, further drainage from both chest drains were minimal and patient improved symptomatically. Patient was minimal and patient improved symptomatically.

Patient was slowly weaned off ventillatory support and extubated after 5 days. Nutritional support was continued through TPN infusion via Right Internal jugular vein CVC and patient remained apyrexial. The patient was shifted to high dependency unit (SITU) after 1 week and finally discharged from the hospital 10 days later.

DISCUSSION: Central venous catheterization is a procedure essentially required for intraoperative, post-operative and critical care including administration of total parental nutrition (TPN). As the number of patients who need long term installation of central venous catheter increase the occurrence of delayed complications - including hydrothorax, hydro mediastinum and cardiac tamponade is also increasing., ${ }^{2,3}$

Hydrothorax is caused by venipuncture of central venous catheter terminal or its placement in the mediastinum. Symptoms of such complications include sudden respiratory distress, tachypnea, central cyanosis, hypotension and tachycardia.

Major factors causing injury to blood vessel wall are the (a) location of the central venous catheter terminal ${ }^{4}$, (b) type of fluids ${ }^{3}$ and(c) the material of the CVC. ${ }^{2}$ 
IN the case scenario presented, the central venous catheter was not mistakenly located during the procedure because there was no difficulty in performing the central venous catheterization, the blood was well aspirated in the catheter and the drug injected through the catheter for induction was efficacious.

In addition, patient symptoms, respiratory distress worsened after induction and intubation due to crystalloid bolus on second day after inserting CVC, indicating delayed bilateral hydrothorax. In cases where the central venous catheter contacts the venous wall or the degree between the blood vessel wall and central venous catheter is larger than $40^{\circ}$, possibility of the punctures is increased by the erosive damage and necrosis of the intima. ${ }^{4}$

Ipsilateral pleural effusion following misplaced catheter tip is not unusual. Possible channels communicating between the peritoneal cavity and the pleural cavity have also been reported. ${ }^{5}$

However, communications between the pleural cavity is rather unusual.

Intravenous nutrition in patient who are unable to start enteral nutrition is a well-established clinical technique. It is also known that the hyperosmotic fluid used for total parenteral nutrition can cause blood vessel puncture through erosive damage. A high caloric count or lipid drugs have the potential to damage the intima because of osmotic pressure difference, which can then cause dislocation of the catheter from the blood vessel through the damaged intima.

The materials of CVC catheter - also have an effect. The risk of thrombus and phlebitis is more with polyurethane and then with poly ethylene. The risk of blood vessel puncture is more common with poly ethylene which is stiffer. In our case, we have used poly urethane catheter but still vessel puncture could have been caused due to hyper tonicity of TPN causing erosive damage to vessel wall, similar to the case reported by Simmons and Henderson in 1991 which led to bilateral pleural and pericardial effusion. ${ }^{6}$

In addition, when trying to aspirate the blood through the CVC in the Intensive Care Unit, blood was aspirated only through the proximal lumen and not the distal lumen. We therefore assumed that the catheter tip could have migrated through the vessel wall to the mediastinum eventually causing bilateral hydrothorax.

We report this case to stress the fact that is very important to verify the abnormal location of the catheter by aspirating blood through the catheter, ${ }^{3}$ take a chest radiograph after the procedure and also check waveforms of the central venous pressure. ${ }^{4}$ Although contrast studies are gold standard for catheter position assessment but it cannot be done routinely.

Newer techniques, such as portable ultrasound devices provides bedside imaging of central veins and detects anatomical variations. ${ }^{7}$ Although there is no doubt that these devices improve the safety of CVC insertion, they may not prevent subsequent malposition or vascular perforation. One important test which could have been done to confirm catheter tip migration is by injecting contrast dye through the catheter and take serial radiographs, which was not done in our case.

Thus, we conclude that catheter tip migration is a recognized phenomenon following central venous catheterization and high degree of clinical suspicion and examination is required for early identification of this dreadful complication to avoid devastating outcome.

\section{REFERENCES:}

1. Myoung Hwa Kim, Dong Jun lee, Mun Chul Kim. Bilateral hydrothorax and cardiac tamponade after right subclavian vein catherization - A case report. Korean J Anesthesiol; 59: S211-7. 
2. Ishibashi H, Ohta K, Ochiai T, Kaisan T, Ishikawa T, Kubota T, et al. Delayed hydrothorax induced by a percutaneous central venous catheter; report of a case. Kyobu Geka. 2002; 55: 213-5.

3. Paw HG. Bilateral pleural effusions: unexpected complication after left internal jugular venous catheterization for total parenteral nutrition. Br J Anaesth.2002; 89: 647-50.

4. Tilak MD, Proctor SM, Donovan JF, Jr, Fitch JC. Extravascular placement of a central venous catheter in the mediastinum. J Cardiothorac Vasc Anesth.2004; 18: 75-7.

5. Kanno T, Yoshikawa D, Tomioka A, Kamijvo T, Yamada k, Goto F. Hydrothorax: an unexpected compication after laparoscopic myomectomy. Anaesthesia 2001; 87: 507-9.

6. Simmons TC, Henderson DR. Bilateral pleural and pericardial effusions because of mediastinal placement of a central venous catheter. JPEN J Parenter Enteral Nutr. 1991; 15: 676-9.

7. Denvus BG, Uretsky BF, Reddy PS. Ultrasound assisted cannulation of the internal jugular vein. A prospective comparison to the external landmarj-guided technique. Circulation 1993; 87: 1557-62.

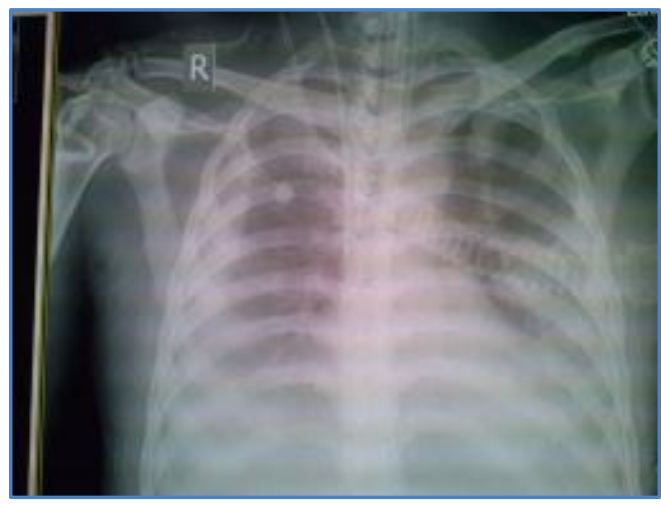

Fig. 1

\section{AUTHORS:}

1. Arpana J.

2. Moses Charles D'souza

3. Kutappa A. M.

4. Deepa Baskaran

\section{PARTICULARS OF CONTRIBUTORS:}

1. Senior Resident, Department of Anaesthesiology and Critical Care, St. Johns Medical College Hospital, Bangalore.

2. Associate Professor, Department of Anaesthesiology and Critical Care, St. Johns Medical College Hospital, Bangalore.

3. Professor and SICU Incharge, Department of Anaesthesiology and Critical Care, St. Johns Medical College Hospital, Bangalore.

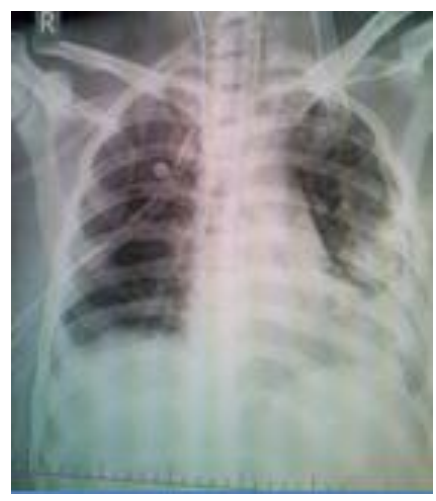

Fig. 2

4. Assistant Professor, Department of Anaesthesiology and Critical Care, St Johns Medical College Hospital, Bangalore.

\section{NAME ADDRESS EMAIL ID OF THE CORRESPONDING AUTHOR:}

Dr. Arpana J,

No. 1928, Nemmadhi, $8^{\text {th }}$, B- Cross, HAL $3^{\text {rd }}$ Stage,

New Thippasandra,

Bangalore-560075.

Email: drarpanak@hotmail.com

Date of Submission: 07/09/2014.

Date of Peer Review: 08/09/2014.

Date of Acceptance: 15/09/2014.

Date of Publishing: 22/09/2014. 